\title{
An Evolutionary Textual Environment
}

\author{
The Unfinished Machine
}

\author{
Manuel Portela
}

\begin{abstract}
This article introduces the notion of evolutionary textual environment as the outcome of a digital experiment. The experiment consisted of transforming a digital archive of Fernando Pessoa's Book of Disquiet into a changing textual space sustained by role-playing interactions. As conceptual and technical artifact, this living archive expresses an innovative model not only for the literary acts of reading, editing and writing, but also for reimagining the book as a network of reconfigurable and dynamic texts, structures, and actions. The programmed features of the LdoD Archive can be used in multiple activities, including leisure reading, study, analysis, advanced research, and creative writing. Through the integration of computational tools in a simulation space, this collaborative archive provides an open exploration of the procedurality of the digital medium itself. The "unfinished machine" metaphor suggests the open-endedness both of the evolving textual environment and of the computational modeling of literary performativity that sustains the whole experiment.
\end{abstract}

\section{A Digital Humanities Experiment}

Can we conceive of models of interface that are genuine instruments for research? That are not merely queries within pre-set data that search and sort according to an immutable agenda? How can we imagine an interface that allows content modeling, intellectual argument, rhetorical engagement?

—Drucker 2013, §34

$\mathrm{T}_{\mathrm{H}}$

HE EXPERIMENT BRIEFly DESCRIBED IN THIS ARTICLE IS AN attempt to answer the challenges posed by textual modeling and interface design according to humanistic principles. ${ }^{1}$ The work known as Fernando

1. The first version of this text was presented at the MLA 2020 convention in Seattle, on January 10, 2020. This panel (382. "Architextures of Knowledge"), which was presided by Jerome J. McGann on behalf of the Society for Textual 
Pessoa's Book of Disquiet has been the point of departure for engaging with "a performative approach to materiality and the design of an interpretative interface" (DRUCKer 2013, §37). As a digital humanities experiment, the $L d o D$ Archive is informed, among other things, by the complexities of poststructuralist critical theories, systems theory, software studies, and materialities of literature. Rather than just a functional artifact or an operational platform - produced according to representational models of literary textuality and engineering principles of transparent human-computer interaction -, it is a conceptual and technical experiment that can be described as an evolutionary textual environment (Portela 2019, 98).

After six years of software development and textual encoding, the $L d o D$ Archive: Collaborative Digital Archive of the Book of Disquiet was finally published, in December 2017, as a free online resource (Portela and Rito Silva 2017, https://ldod.uc.pt/). At once textual archive and textual machine, the multifunctional and multilayered digital artifact that we published on the web had evolved into something quite different from what I had been able to conceive when the first ideas were set to paper. In April 2009, I wrote down for the first time the title for what would turn out to be a decade-long research project: "No Problem Has a Solution: A Digital Archive of the Book of Disquiet". The phrase "No Problem Has a Solution" is the English translation of the incipit from one of the fragments belonging to Fernando Pessoa's Livro do Desassossego [Book of Disquiet]. I came to the project title entirely by chance: a series of autograph notebooks by Pessoa had just been made freely available, as digital facsimile images, through the Portuguese National Library digital collection. After searching for "L. do D." within the metadada for those notebooks, I discovered the facsimile of notebook $144 \mathrm{D}(2)$, and then browsed it until I came to folio $135 \mathrm{r}$, and read the handwritten text dated 18-7-1916 (see Fig. 1):

No problem has a solution. None of us can untie the Gordian knot; either we give up or we cut it. We brusquely resolve intellectual problems with our feelings, either because we're tired of thinking, or because we're afraid to draw conclusions, or because of an inexplicable need to latch on to something, or because of a gregarious impulse to return to other people and to life. Since we can never know all the factors that a problem entails, we can never solve it.

Scholarship, also included Marta L. Werner ("Editing Dickinson's 'Master' Documents in a New Hour") and Ryan Cordell ("Speculative Bibliography"). I am grateful for their comments. I would also like to acknowledge comments made by the audience during the discussion. 
To arrive at the truth we would need more data, along with the intellectual resources for exhaustively interpreting the data.

(Zenith 2002, Text 3332)

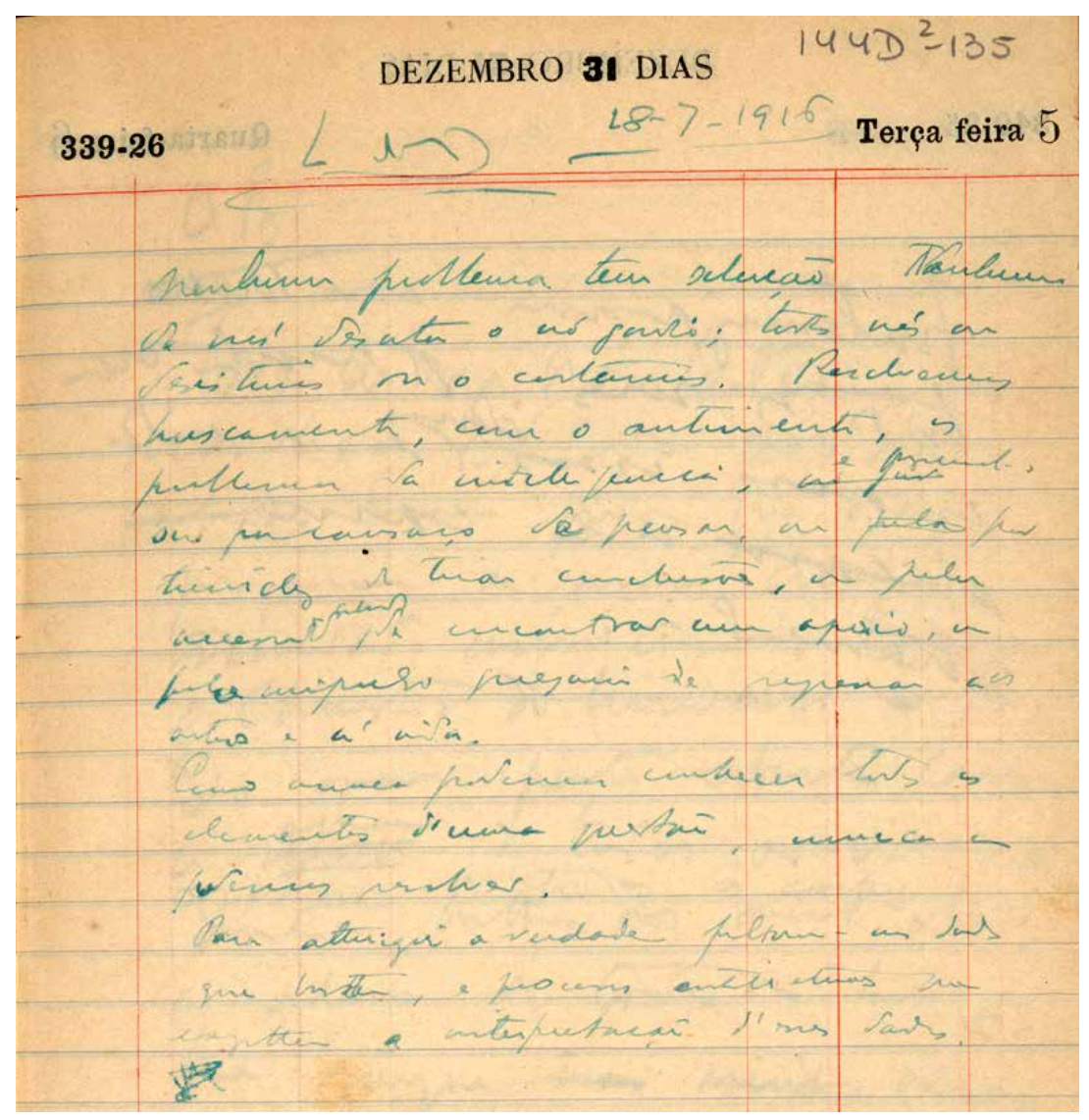

Figure 1. Autograph manuscript containing a piece for the Book of Disquiet, included in notebook 144D(2) -135r. "L. do D. 18-7-1916 Nenhum problema tem solução". BN reference for the digital facsimile: bn-acpc-e-e3-144d2_0351_135r_t24-C-R0150. (C) National Library of Portugal. Reprinted with permission.

Pessoa's reflection (or rather, the reflection of the first heteronym he created for this particular work) about the impossibility of pursuing a course of

2. Texts in Richard Zenith's English E-PUB edition will be referenced by their number (not by page number). 
inquiry to its ultimate consequences, and the somewhat uncanny use of the notion of data interpretation, resonated with my own sense that digital mediation of textual processes offered unchartered possibilities asking for further conceptual and technical exploration. So this textual fragment was unconsciously invested with an allegorical and speculative significance for the entire enterprise: it was both a material instance of textual processes in the Book of Disquiet whose digital remediation posed challenging intellectual problems, and a philosophical comment on the impossibility of thinking problems through towards some satisfactory solution.

Somehow, the phrase "no problem has a solution" implied the need for an entirely open-ended artefactual inquiry into the nature of reading, editing, writing and digital materiality which could use the textual space of this particular work to produce a more general model of literary action. Or, in another equally appropriate implication, the inevitability of failure. Given the technical and conceptual difficulties I anticipated and given the uncertain outcome of our efforts, the building of this archive could turn out to be a generator of unsolvable problems. Besides, the co-occurrence of the tokens "problem", "solution", and "data" provided yet another layer of meaning, in which this piece of text could be read in parodic relation to the typical vocabulary of software engineering. Its resonance thus extended from the material level of autograph inscription to the philosophical and literary level of meaning production, and to the methodological level of computational parsing and processing. Which were the problems that could only not be solved?

My interest in digital editing had started in the early 2000s when I began looking at and working with various digital literary archives that were under development since the mid-1990s. Those early projects were addressing the problem of reediting literary works by adapting established principles of critical and documentary editing in print media to the hypertext and hypermedia capabilities of the networked computer. Computational advances were changing the technologies and practices of textual production and reproduction. Digital editors used those changes not only for migrating the archive of literary production to the new medium, but also for testing forms of textual encoding made according to specific editorial theories, such as genetic criticism or social editing. Although their initial focus was on making accessible and linkable many different types of source materials in verbal and visual media, research gradually shifted to explore other possibilities created by the machine-readability of digitized texts and, later, by the collaborative and socialized processes of the evolving ecology of the web. 
It was within this technical and intellectual context that I began to look for a work that would sustain a set of relevant literary interrogations related to material and social processes of textual mediation. As an unfinished modular work composed of an open set of a few hundred witnesses in various stages of completion, the Book of Disquiet seemed the most likely candidate for making a new experiment in digital critical editing. Its modular and fragmentary structure matched the modular nature of digital objects. Furthermore, the Book of Disquiet is a major literary achievement of modernist awareness of the self, representative of Pessoa's writing practice and of European modernism, and it continues to interest all kinds of contemporary readers. It is the most often quoted text by Pessoa on the internet, as well as his most translated work worldwide. Material form and literary significance thus came together in justifying this choice.

When I started working on the project, it was mostly as a metaeditorial representation of four versions of the Book of Disquiet, which would allow interactors to automatically compare them against the autograph sources and against each other at the micro-scale of textual transcription and at the macro-scale of bibliographic structure. The focus would be placed on representing the processuality of editing rather than making a claim for an improved, definitive or final edition of the work. The archive would be data modeled in ways that would give us a sense of the authorial and social processes of textual production that resulted in several versions of the work. This was the version of the project that was written into the original February 2011 application for funding. ${ }^{3}$

Once we started working, in March 2012, the concept of the archive gradually morphed into the concept of the dynamic archive and, soon after that, of the literary simulator (Portela 2017). By early 2013 all of the dynamic functionalities had subsumed the initial archival metaeditorial logic, which by then had been reconceived as one set of functions among several other functionalities to be programmed into the ongoing literary machine. For five years we continued to develop both the critical editing

3. This project was selected for funding by the Portuguese national research agency (FCT - Foundation for Science and Technology), and the LdoD Archive was developed by a team of literary and computer researchers between 2012 and 2017. The first release of the $L d o D$ Archive was launched on December 14, 2017. New content and new functionalities have been developed since then. Future releases with added layers of virtualization, simulation and analysis are scheduled for the coming years. The source code of the LdoD Archive is available here: https://github.com/socialsoftware/edition. For a detailed bibliography of the project see: https://ldod.uc.pt/about/articles/?lang=en. 
and metaediting component, on the one hand, and the interactive simulation component, on the other. Each of those components was refined and integrated into a common textual space which was intended to model literary performativity beyond the bibliographic horizon. The Book of Disquiet thus became a digital literary space for probing textual processuality in general. It was as if the data processing of this particular work had opened up the entire field of literary performance. ${ }^{4}$

The notions of literary simulation and evolutionary textual environment were unintended creative outcomes of this project, two artefactual embodiments of the intersection between scholarly practice and literary imagination. Conceived as a metaeditorial archive containing several editorial versions of Fernando Pessoa's unfinished work Book of Disquiet, the project evolved into a new kind of conceptual and technical artifact, which I now see, in retrospect, as both a scientific and literary invention. Many threads of ideas have fed into this invention, among which I highlight the following: the hypertext rationale for electronic editing as theorized and put to practice by Jerome McGann and other digital critical editors in the 1990s and 2000s (McGANN 2001, 2006); the notion of performative materiality as expressed in Johanna Drucker's analyses of codependence between material form and interpretative action (DrUCKER 2009); John Cayley's creative and critical explorations of programmability and reading, recently synthesized in the concept of grammalepsy (CAYLEY 2018).

The notion of evolutionary textual environment refers to its evolving structure and functionalities as the researchers continue to experiment with what is possible in this medium, on one hand, and to the changes in its content and uses as interactors perform their various actions according to a role-playing rationale, on the other. The dynamic and socialized functions of the $L d o D$ Archive offer an interpretative interface through which subjects are asked to see how their actions are constitutive of the textual environment itself. In this reading-editing-writing space, the conditions of production and reception of the web have been integrated into the ecology of its literary and computational form. The $L d o D$ Archive changes its content and the relations among its elements as interventions by this community of users modify the configuration of its textual space over time.

Not only are interactors invited to see the processuality of reading, editing, and writing as it is being presented from multiple perspectives through the $L d o D$ Archive's contents and functions, but they can also experiment with their own acts of reading, editing, and writing by inscribing them into the system. The system evolves as those inscriptions and its analyses

4. A general theory of the $L d o D$ Archive can be found in Portela 2022. 
of the interactions are iteratively used for changing and adapting its own affordances and constraints. The double rationale of our modeling of literary performativity - as both representation and simulation - generates an evolutionary textual environment, whose content and structure evolve to register the interventions of its interactors and foster their creativity.

This evolutionary textual environment is the unanticipated result of the attempt to offer a theoretical and computational answer to the question "what is the Book of Disquiet?" The project was triggered by the initial desire to model the relation between two answers to that question: first, it is an unfinished work, composed of a semi-determined set of modular texts in various stages of composition; secondly, it is also a set of specific editions that have used diverse criteria for transcription, selection and organization of their texts. Once we began to model the relation between those two perspectives, our focus shifted from representing the actuality of those relations as documented in the work's authorial and editorial archive to simulating the processes through which a work becomes a work. These processes were referred to, in the redesigned model, as literary performativity, by which I mean the set of material and social processes that sustain the production of literary meanings, forms and experiences. These processes have been abstracted as reader-function, editor-function, author-function

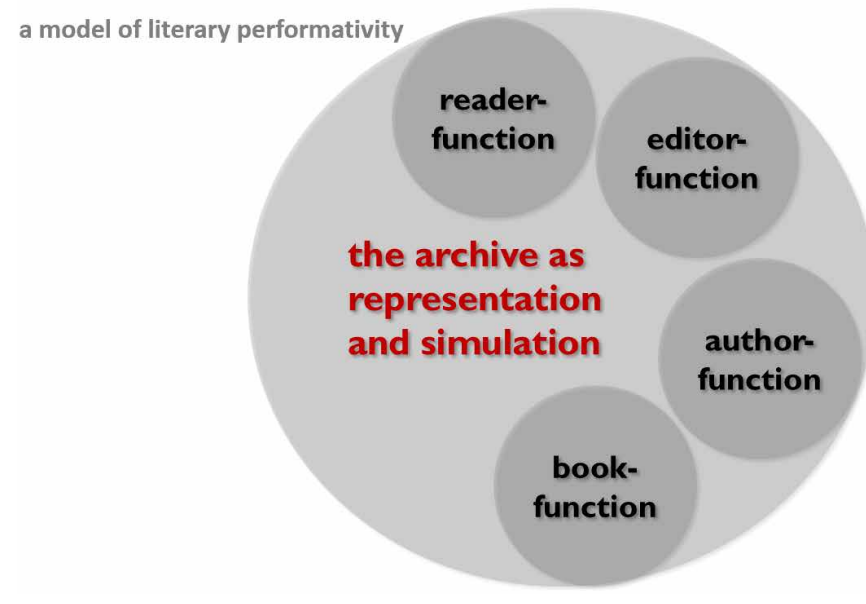

Figure 2. Literary performativity as a network of role-playing actions.

and book-function (see Fig. 2). 
The $L d o D$ Archive gradually morphed into a far more complex imaginary creature, whose main goal is to develop a data model and a series of functionalities that are able to express the relation between a representation layer and a simulation layer in ways that address an additional set of fundamental questions: how can we represent and simulate the processuality of the book under construction (as both material production and conceptual operator)? And how can we represent and simulate the processuality of the acts involved in its construction, such as reading, editing, and writing? Each of those dimensions of performativity are modeled as representations: writing acts by encoding the Book of Disquiet as set of autograph materials; editing acts by encoding the Book of Disquiet as set of expert editions of

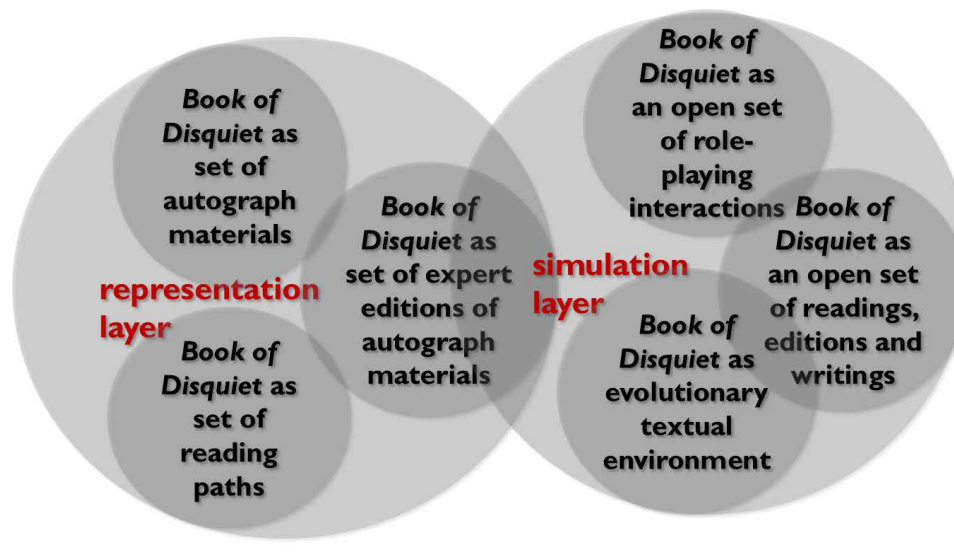

Figure 3. Layers of representation and simulation in the LdoD Archive.

autograph materials; reading acts by showing the Book of Disquiet as set of actual reading paths.

At the same time, the simulation of those actions as a range of possibilities requires them to be experienced through interventions on the textual materials that feedback onto the representational layer (see Fig. 3). Modeled through this simulation layer, the Book of Disquiet within the LdoD Archive is transformed into an open set of role-playing interactions - that is, as an open set of readings, editions, and writings - and, ultimately, as an evolutionary textual environment (including its own source code). Insofar as the contents and structure of the system respond to the ongoing actions of its subjects, the processuality and performativity of those literary actions are experienced as constitutive of the system. Given that reading, editing, and writing have been conceived to explore a wide range of human-only, 
machine-assisted, human-assisted, and machine-only interactions, the model can also be understood as an experimental digital environment in which a large suite of digital tools can be adapted, used and transformed (see Fig. 4).

an experimental digital environment

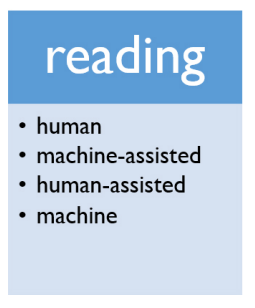

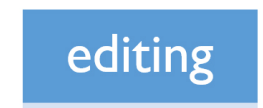

- human

- machine-assisted

- human-assisted

- machine

\section{writing}

- human

- machine-assisted

- human-assisted

- machine

Figure 4. From manual-only to fully algorithmic: an experimentation with the digital medium.

Interactors experience their actions not only as a series of textual possibilities that emerge from a range of predefined values and parameters, but also as an open exploration of literary performativity itself. According to its simulation rationale, each output is the result of a collaborative action in which the algorithmic production of the system is modified by intentions and procedures of the interactor. Outputs - whether taking the form of

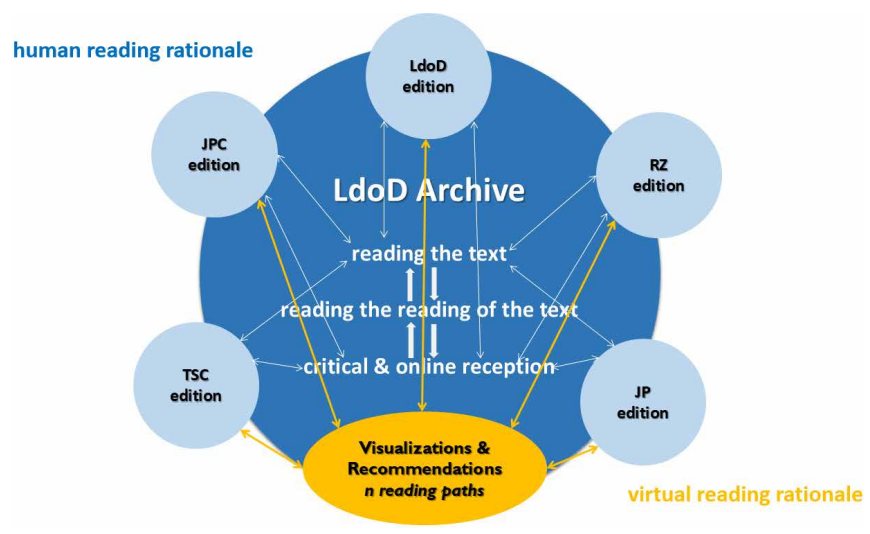

Figure 5. From human-assisted and machine-assisted human reading to human-assisted and machine-assisted virtual reading. 
reading trails, edited sequences, classification taxonomies, macro-visualizations or new texts - are not entirely determined by the system's internal logic since they will capture the human processing of its programmed processing.

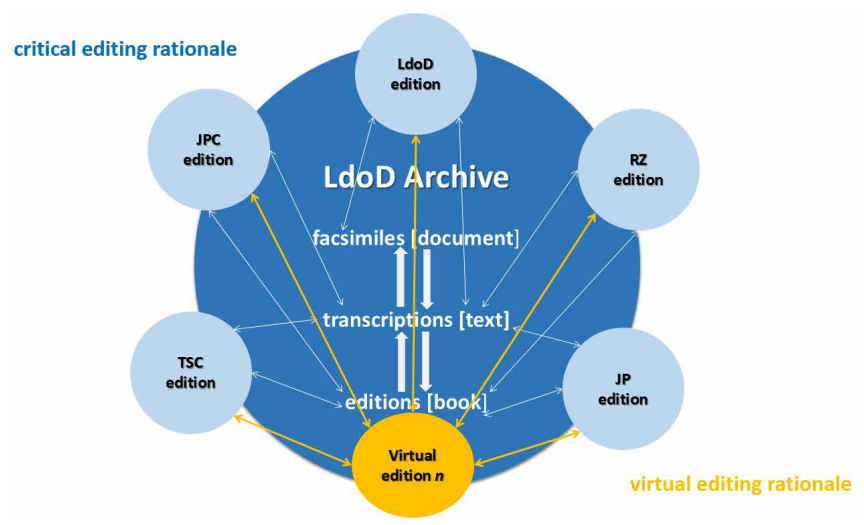

Figure 6. From human-assisted and machine-assisted critical editing to human-assisted and machine-assisted virtual editing.

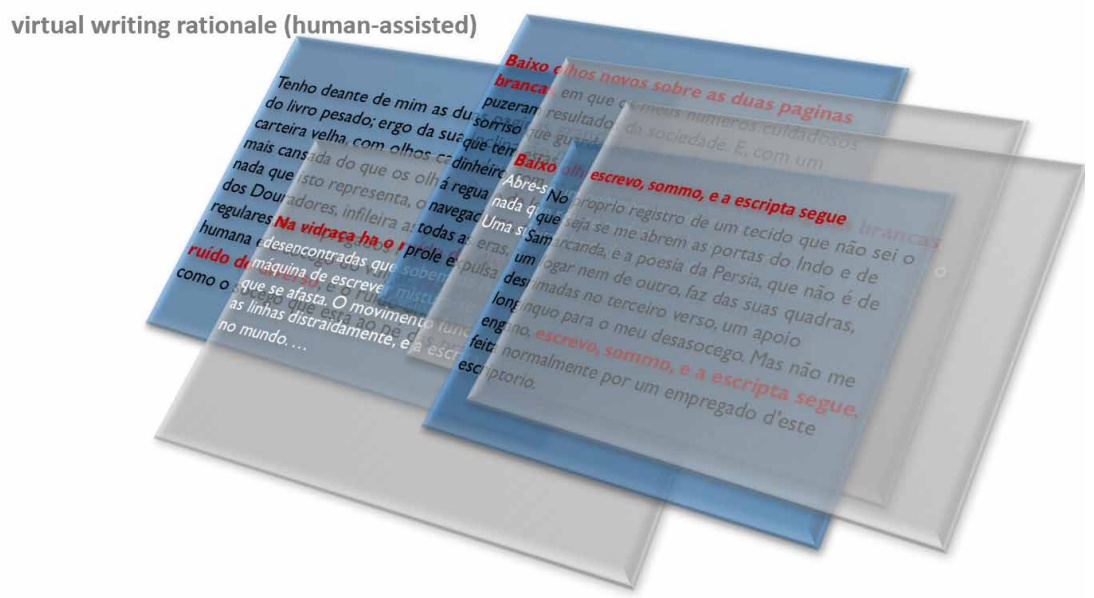

Figure 7. Human-assisted virtual writing. 


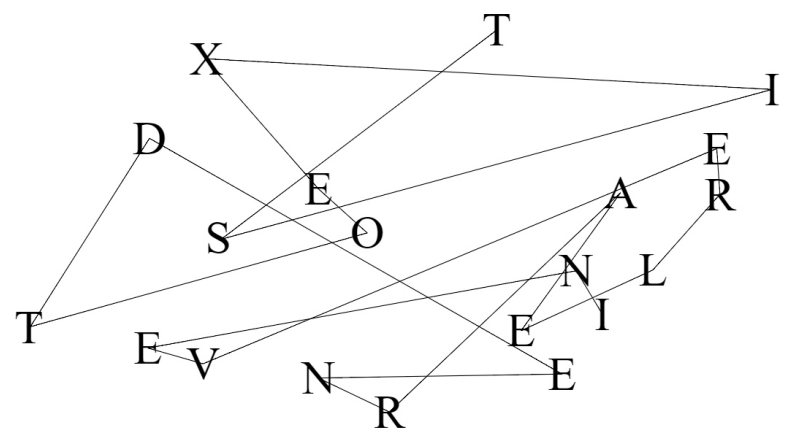

TP01
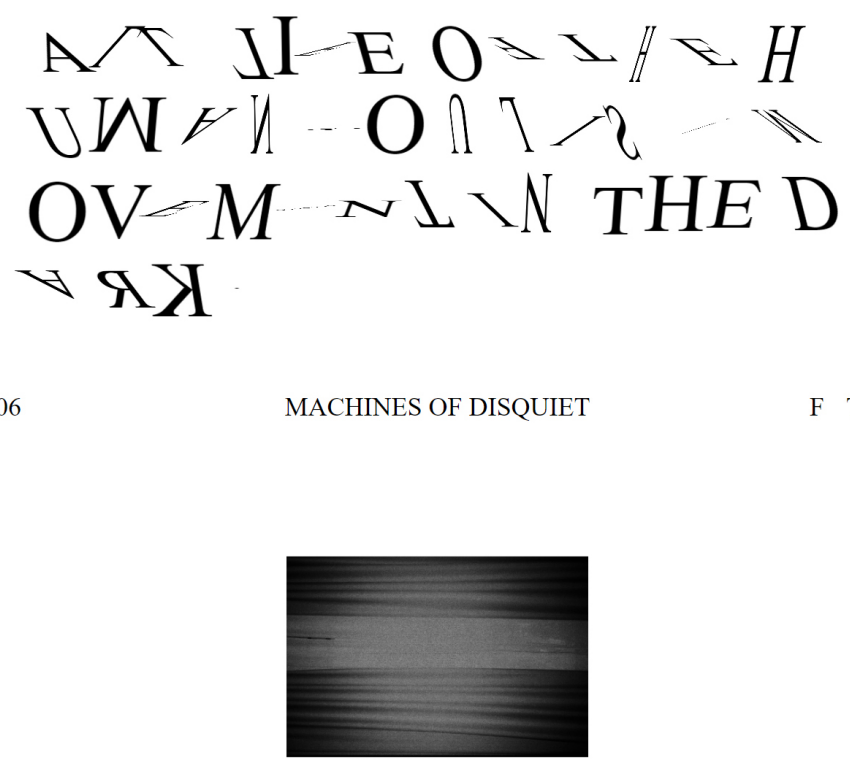

We get tired of everything except of understanding.

Figure 8. Machine-assisted virtual writing. Luís Lucas Pereira, Machines of Disquiet (2014-2018). http://mofd.dei.uc.pt/ 
This openness of the $L d o D$ Archive to the interpretative action of its users takes place at two entangled levels. On one level, interpretative action happens according to flexible and shifting perspectives on its textual representations. On another level, interpretative action works according to game-like practices in role-playing simulations (see Figs. 5, 6, 7, and 8). As users inscribe their reading, editing and writing acts, the LdoD Archive documents the results of those interactions and offers them for further reading, editing, and writing acts, and further analysis. Both user-created content and reflexive textual analyses are part of the evolutionary textual environment. The evolutionary textual environment itself could be redescribed as an evolving socialized literary practice in networked programmable media. Its fusion of technical and conceptual model, on one hand, with actual acts of reading, editing, and writing, on the other, bring into being the unbounded and iterative nature of the textual condition.

If we think of it in ethical terms, we could say that the $L d o D$ Archive is not a system for automating literary production, reception, or analysis. In this respect, it sets itself apart from dominant engineering approaches to computational creativity in artificial intelligence and from dominant digital humanities approaches to textual processing. Rather, its ecology of machine-assisted human action and human-assisted machine action turns algorithmic processes into literary procedures for opening up textual spaces to critical and creative explorations. The result is an evolutionary textual environment fed by the unpredictability and creativity of human interactors in a live, time-distributed, and collaborative social process. Its purpose is to instantiate the conditions that allow the reiteration of the relation between potentiality and actuality for each individual role-playing action. Given that this complex computational environment originated in an attempt to model the processuality of bringing a book into existence (in this particular case the Book of Disquiet by Fernando Pessoa), we can say that the bibliographical imagination itself is reconfigured as a linguistic and physical force in the dynamics that produces the literary as material poetics and social semiotic practice. 


\section{Modeling Literary Performativity}

And I offer you this book because I know it is beautiful and useless. It teaches nothing, inspires no faith, and stirs no feeling. A mere stream that flows towards an abyss of ashes scattered by the wind, neither helping nor harming the soil.

—Book of Disquiet, "Perystile", c. 1913, transl. Zenith

The Book of Disquiet contains many references to reading, writing, and books, including self-references to the acts of reading, writing, and organizing "this book". Images of reading and images of writing are used to characterize the writing self as the empty being of fictional imagination: "As my feet wander I inwardly skim, without reading, a book of text interspersed with swift images, from which I leisurely form an idea that's never completed" (Zenith 2002, Text 181). Describing the self as "a book of text interspersed with swift images", this passage links reading-writing and self-creation with the imagination of the book. The swiftly skimmed book is an image for the inscrutability of the self, but also for the shifting projective nature of the book. The "idea that's never completed" suggests the incompleteness of self-production through reading-writing feedbacks, but also the ongoing process of creating and perceiving form in literary acts.

In the $L d o D$ Archive, processuality has been modeled through the notion of literary performativity. Our aim is to engage the constructedness of literary experience as a shared social practice dependent upon acts of reading, writing, and editing. In this performative space, programmed affordances and constraints enable subjects to move across different positions in its field of literary action: from reading to editing, from reading to writing, from editing to reading, from editing to writing, from writing to reading, and from writing to editing (see Fig. 9). Interactors are asked not only to observe a parametrized ensemble of reading, editing, and writing forms and relations, but to reconstitute those forms and relations by performing according to variable positions in the field. The "idea that's never completed" and the "insolvable problem" can be understood as metaphors for the $L d o D$ Archive's attempt to represent and simulate processuality as an open-ended unfinished (and unfinishable) machine. 


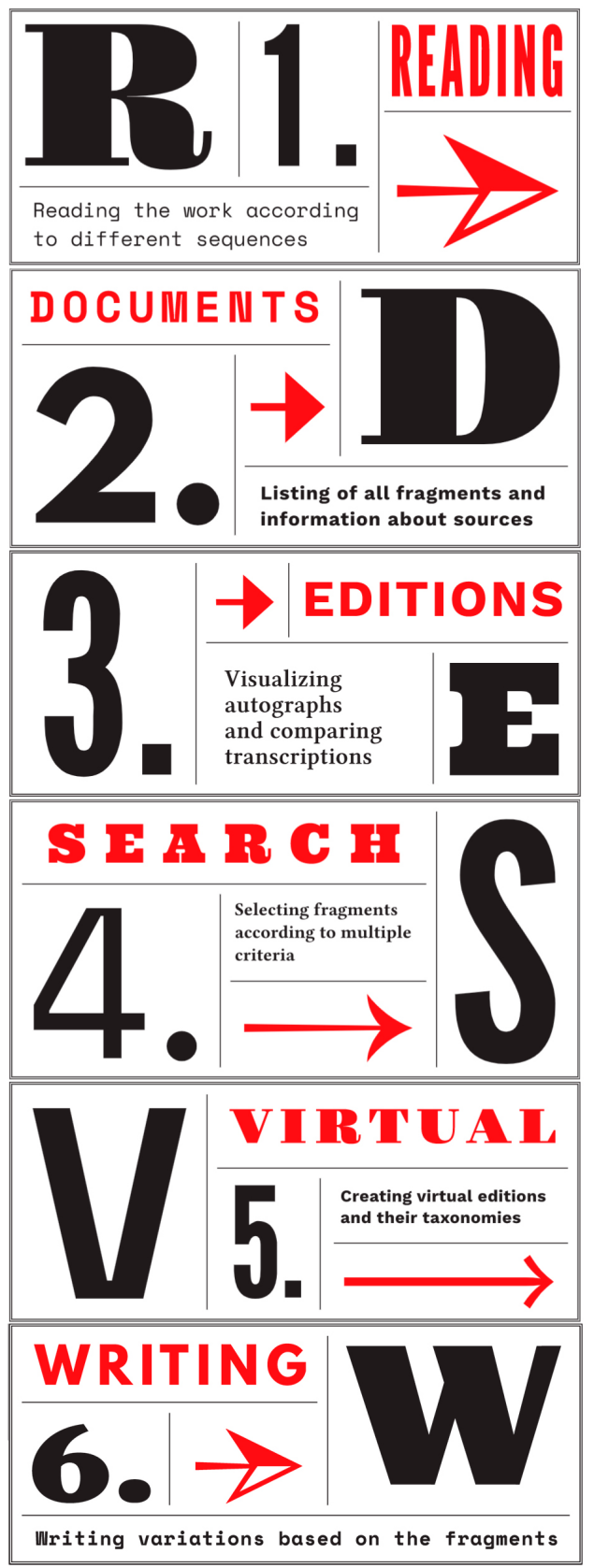

Figure 9. Shifting positions and changing interfaces in the LdoD Archive: Reading, Documents, Editions, Virtual, Search, and Writing. 
The Book of Disquiet itself offers several examples of position shifts which can be equated with the role-playing rationale of the $L d o D$ Archive. For example, the shift from reading to writing instantiates the relation of reader-function to author-function:

I know no pleasure like that of books, and I read very little. Books are introductions to dreams, and no introductions are necessary for one who freely and naturally enters into conversation with them. I've never been able to lose myself in a book; as I'm reading, the commentary of my intellect or imagination has always hindered the narrative flow. After a few minutes it's I who am writing, and what I write is nowhere to be found.

(Zenith 2002, Text 417)

Similarly, the change from reading to editing expresses the relation of reader-function to editor-function:

In one of those spells of sleepless somnolence when we intelligently amuse ourselves without the intelligence, I reread some of the pages that together will form my book of random impressions. And they give off, like a familiar smell, an arid impression of monotony. Even while saying that I'm always different, I feel that I've always said the same thing; that I resemble myself more than I'd like to admit; that, when the books are balanced, I've had neither the joy of winning nor the emotion of losing. I'm the absence of a balance of myself, the lack of a natural equilibrium, and this weakens and distresses me.

(Zenith 2002, Text 442)

The shift from writing to reading can be equated with the movement from author-function to reader-function:

In the faint shadows cast by the last light before evening gives way to night, I like to roam unthinkingly through what the city is changing into, and I walk as if nothing had a cure. I carry with me a vague sadness that's pleasant to my imagination, less so to my senses. As my feet wander I inwardly skim, without reading, a book of text interspersed with swift images, from which I leisurely form an idea that's never completed.

(Zenith 2002, Text 181)

The split between embodied self as phenomenological entity and fictional self as an imaginary character constituted by written language is at 
the heart of Pessoa's account of literary depersonalization. In "The Art of Effective Dreaming for Metaphysical Minds", depersonalization is divided into three stages, of which the last one leads to the ability to be several selves at the same time: "The highest stage of dreaming is when, having created a picture with various figures whose lives we live all at the same time, we are jointly and interactively all of those souls" (ZeNith 2002, Text 495). This multiplication of being is manifest in the proliferation of heteronyms or writing selves:

We are mere ashes endowed with a soul but no form - not even that of water, which adopts the shape of the vessel that holds it. With this $\square$ thoroughly established, complete and autonomous plays can unfold in us line by line. We may no longer have the energy to write them, but that won't be necessary. We'll be able to create secondhand; we can imagine one poet writing in us in one way, while another poet will write in a different way. I, having refined this skill to a considerable degree, can write in countlessly different ways, all of them original. ${ }^{5}$

(Zenith 2002, Text 495)

Writing selves appear as fictional authors and become independent of Pessoa's self by virtue of their way of writing. Pessoa's fictionalization of heteronymic writing may also be equated with the author-function. The act of writing "in a different way" constitutes the heteronymic author as an emergent function of a specific writing practice (including each heteronym's projected books). In turn, Pessoa's role, in this meta-literary system, is to perform as the author-producing author, i.e., as a second-degree manifestation of the author-function.

The production of the book in the Book of Disquiet contains material evidence of the performativity of literary action that we have tried to model in the LdoD Archive. Additionally, there are more than a dozen external references to the Book of Disquiet in letters to friends, notes, title lists, and plans for publication. Imagining this particular book through selfdescription is only one instance of the continuing imagination of books as expressions of his various heteronyms, on the one hand, and as expressions of Pessoa's meta-authorial condition on the other. Their existence as fictional authors, as well as his existence as author of authors, depend upon

5. Pessoa sometimes leaves blank spaces for one or more words as if he intended to come back later to that point and fill in the missing words. Here, the $\square$ is the symbol used by the editor to mark these blank spaces in the manuscript. 
the complex workings of the book-function as a constitutive element of literary performativity. At once assemblage of written discourse in search of conceptual and material unity, on one hand, and imaginary horizon for each new act of writing, on the other, the book-function is both a physical and metaphysical operator.

The incompleteness of the Book of Disquiet as a book in progress reincarnates in the processuality of reading, editing, and writing as acts of inscription of consciousness in the duration of time. Appropriating the time-based nature of digital media, the $L d o D$ Archive places representation in a dynamic and complex relation with simulation. Beyond the archival representation of the unique and historical occurrence of a set of texts in several stages of completion, it attempts to model and experiment with the flows of reading, editing, and writing that constitute and maintain a literary space through the literary imagination of the book. The flexibility of the digital inscription is used to explore the potentiality of those actions as an iterative ongoing process, and the material and conceptual operator we designate as book, which has become one of the main producers of the literary as a set of practices of evocation and intensification of the human experience of the world.

The performativity of reading is expressed as (1) multicursal visualization of reading paths, (2) analysis of specific reading practices (such as the history of the Book of Disquiet's expert critical reception or its current social media reception), and (3) reading of one's own reading trails. The performativity of editing enables interactors to (1) compare authorial witnesses against their various transcriptions, (2) compare expert editions against each other, and (3) produce their own editions (including annotations and taxonomies) using both manual and computer-assisted processes. The performativity of writing takes the form of (1) exploration of autographic writing processes, (2) new writing acts anchored on specific passages, (3) new writing acts based on machine-assisted procedures and constraints. Each process (reading the book, editing the book, and writing the book) can be experienced in relation to other representations or simulations of itself, and also in relation to all other processes.

The LdoD Archive may, ultimately, be described as a conceptual, material, and technical experiment that attempts to simulate literary functions as a dynamic field of discursive interactions for imagining our being in the world through written language. Electronic remediation of Pessoa's text has been reimagined as a meta-reading, meta-editing, and meta-writing exercise that allows interactors to play with and investigate the social dynamics of textual production. They are invited to engage reading, editing, and 
writing as performative actions that constitute and sustain a literary field. The problem of writing, editing, and reading a book about the inner existence of a written self becomes a material experiment with the potentiality of literary imagination that takes advantage of the procedural and collaborative affordances of the medium.

Virtualizing the Book of Disquiet in a dynamic archive is our way of modeling the literary performance for the current environment of networked reading, editing, and writing spaces. This implies reimagining the dynamics between editing and the codex, and between reading and writing in ways that fully engage the possibilities and constraints of the digital medium, including its diverse layers of encoding, programmability, visualization, and interaction. The Book of Disquiet is the ideal work for an attempt at reimagining the textual condition in ways that fully explore the computer as an expressive medium. To engage reading, editing, and writing - inside and outside bibliographic structures - through performative simulations is our material exploration of the medium's processing and participatory affordances for literary potentiality.

University of Coimbra

\section{Works Cited}

CAyley, John. 2018. Grammalepsy: Essays on Digital Language Art. London: Bloomsbury.

Drucker, Johanna. 2009. SpecLab: Digital Aesthetics and Projects in Speculative Computing. Chicago: University of Chicago Press.

- 2013. "Performative Materiality and Theoretical Approaches to Interface". Digital Humanities Quarterly 7.1: § 1-43. http://digitalhumanities.org:8081/dhq/ vol/7/1/000143/000143.html

McGann, Jerome. [1996] 2001. "The Rationale of Hypertext”. In Radiant Textuality: Literature after the World Wide Web, 53-74. New York: Palgrave Macmillan.

2006. "From Text to Work: Digital Tools and the Emergence of the Social Text". Text 16: 49-62.

Pereir a, Luís Lucas. 2014-2018. Machines of Disquiet. Coimbra: Centre for Informatics and Systems of the University of Coimbra. http://mofd.dei.uc.pt/

Portela, Manuel. 2017. "The Book of Disquiet Archive as a Collaborative Textual Environment: From Digital Archive to Digital Simulator". In The Writing Platform: Digital Knowledge for Writers. Brisbane: Queensland University of Technology. http://thewritingplatform.com/2017/07/book-disquiet-archive-collaborative-textual-environment-digital-archive-digital-simulator/ 
2019. "The LdoD Archive as a creative textual environment and a model of literary performativity”. In Computational Creativity Meets Digital Literary Studies, Dagstuhl Reports, 9.4: 87-106, edited by Tarek Richard Besold, Pablo Gervás, Evelyn Gius, and Sarah Schulz.

- 2022. Literary Simulation and the Digital Humanities: Reading, Editing, Writing. New York: Bloomsbury [forthcoming].

Portela, Manuel, and António Rito Silva, eds. 2017-2021. LdoD Archive: Collaborative Digital Archive of the Book of Disquiet. Coimbra: Centre for Portuguese Literature at the University of Coimbra. https://ldod.uc.pt/

Zenith, Richard, ed. 2002. The Book of Disquiet, by Fernando PessoA, translated by Richard Zenith. London: Penguin Books. E-PUB. 